\title{
$\angle S$ Research Square

\section{Effectiveness of Combined Tui-Na and Oral Chinese Medicine on Knee Osteoarthritis - a Randomized Wait-List Controlled Trial Study Protocol}

\section{Kin Ho Chan \\ Yan Oi Tong \\ Jessica YL Ching}

The Chinese University of Hong Kong Faculty of Medicine

Kam Leung Chan

The Chinese University of Hong Kong

Hoi Yi Lau

Yan Oi Tong

Ka Man Chu

Yan Oi Tong

Kenny Chan

Yan Oi Tong

Hon Fai Pang

Yan Oi Tong

Tianhe Song

The Chinese University of Hong Kong

Bacon Fung Leung $\mathrm{Ng}$

HA: Hospital Authority

Zhi-Xiu LIN ( $\nabla$ linzx@cuhk.edu.hk)

Chinese University of Hong Kong https://orcid.org/0000-0001-8635-0658

\section{Study Protocol}

Keywords: Knee Osteoarthritis, Knee Pain, Tui-Na, Chinese Herbal Medicine, Randomized Wait-List Controlled Trial

Posted Date: December 31st, 2020

DOI: https://doi.org/10.21203/rs.3.rs-136364/v1

License: (c) (1) This work is licensed under a Creative Commons Attribution 4.0 International License.

Read Full License 


\section{Abstract}

\section{Background}

Knee osteoarthritis (KOA) is a common degenerative joint condition that causes disability and pain amongst the elderly. The prevalence of KOA among persons aged 63 or above is approximately $30 \%$. It is estimated that around $10 \%$ of the Hong Kong residents will have KOA by the year 2036. Previous studies have reported the positive effect of Tui-na treatment and Chinese herbal formula Du-Huo-Ji-Sheng Decoction (DHJSD) for KOA treatment. However, the therapeutic effect of the combination of both methods is unknown. The current study aims to evaluate the therapeutic effect of the combined therapy of Tui-na and oral administration of DHJSD on KOA using a randomized controlled design.

Methods

This study protocol describes a prospective, randomized wait-list controlled trial which will be conducted in the Yan Oi Tong Chinese Medicine Clinic cum Training and Research Centres (in Tuen Mun and Islands Districts). The eligible subjects will be randomly assigned to the treatment group and wait-list control group in a 1:1 ratio. Both the treatment and wait-list control groups will receive 8 sessions of Tui-na manipulation. DHJSD will be given to the subjects in the treatment group but not the wait-list group. The primary outcome will be assessed using the WOMAC score at 4 weeks after randomization. The secondary outcomes include the WOMAC scores at 2 weeks and 8 weeks after randomization and the health-related quality of life, the 5-level EQ-5D version (EQ-5D-5L), which will be conducted at 2 and 4 weeks after randomization and at follow-up (8 weeks after randomization).

\section{Participants}

Seventy subjects aged 50 years or above, who meet the criteria of KOA according to the American College of Rheumatology, with Western Ontario and McMaster University Osteoarthritis Index (WOMAC) score $\geq$ 39 will be recruited.

\section{Discussion}

The study will provide clinical evidence for the effectiveness of the combined Tui-na and oral administration of DHJSD for the treatment of subjects with KOA. The study will also provide information for large-scale randomized placebo-controlled trial in the future.

Trial registration

ClinicalTrials.gov, ID: NCT04492670. Registered on 28 July 2020.

\section{Background}


Knee osteoarthritis (KOA) is a common degenerative joint condition and one of the most common causes of disability and pain among the elderly. The prevalence of KOA among persons aged 63 or above is approximately $30 \%$, and this figure increases with age [1]. It is predicted that $31 \%$ of Hong Kong residents will be aged 65 or above by the year 2036 [2], meaning an estimated 10\% of Hong Kong residents will be suffering from KOA at the time. Since the morbidity rate of KOA is extremely high, it is imperative to establish an effective treatment method for KOA.

The effectiveness of conventional therapies for KOA is limited for many reasons, including an array of adverse side effects and drug resistance from the use of approved pharmacological drugs [3,4]. Currently, the ultimate solution is surgery, but it is usually with exceptionally prolonged waiting periods. The effectiveness of Total Knee Replacement (TKR) makes this option unfavorable (why?? Please rewrite this sentence.). According to the database, the waiting time for total knee replacement (TKR) is approximately 25 to 107 months in Hong Kong [5], and the probability of having the postsurgical pain of TKR is approximately $20 \%$ [6].

Historically, Chinese medicine techniques such as Tui-na have been extensively used to treat KOA, and the methods have been refined. The effect of Tui-na is well studied and widely reported [references??]. Tui-na therapy can relieve the symptoms of KOA due to its ability to increase the local blood circulation in the affected muscles [7]. Moreover, it has fewer adverse effects than other treatment methods and is generally well accepted by the KOA patients.

Despite the effectiveness of Tui-na therapy, the number of qualified Tui-na practitioners in Hong Kong is limited. Currently, there are 18 clinics that are supported by the Hospital Authority and operated by Nongovernmental organizations (NGOs), only 13 of them provide Tui-na services. On average, a Tui-na practitioner is able to attend to approximately 10 patients per day. Since Hong Kong can only provide limited Tui-na services to KOA patients, we aim to maximize the effectiveness of the Tui-na by adding other therapies to create a synergism on the treatment plan. This is expected to reduce the number and frequency of Tui-na sessions required for KOA patients. Du-Huo-Ji-Sheng Decoction (DHJSD) is a widely used Chinese herbal formula for the treatment of arthritis in China $[8,9]$. It has been shown that DHJSD could improve clinical symptoms, knee function and quality of life for patients with KOA by inhibiting cartilage apoptosis (please check this. Usually apoptosis refers to cell death, and not to tissue damage.) [10]. In addition, DHJSD treatment for KOA showed a lower risk of adverse events than standard western treatments [11]. Moreover, DHJSD has been demonstrated to have significant anti-inflammatory effects through promoting lymphatic drainage function in animal studies [12]. Yet the therapeutic efficacy of the combination of Tui-na and oral administration of DHJSD remains unknown. The present proposed project aims to evaluate the effectiveness and safety of a combination of Tui-na and oral administration of DHJSD for the treatment of KOA.

In this study we will use the Western Ontario and McMaster University Osteoarthritis Index (WOMAC) $[13,14]$ as the primary outcome measure. WOMAC, which consists of a set of 24 questions, is widely used among health professionals to evaluate the patients with osteoarthritis. The WOMAC is obtained from the 
sum of all 24 individual scores, and a score of 39 and above indicate that the patients suffer from severe osteoarthritis [15]. The WOMAC Cantonese version has been validated and is available upon requested [16]. Besides, the EQ-5D-5L $[17,18]$ will be used to assess health-related quality of life, particularly the utility values. The EQ-5D-5L essentially consists of 2 pages: the EQ-5D descriptive system and the EQ visual analogue scale (EQ VAS). The descriptive system comprises five dimensions: mobility, self-care, usual activities, pain/discomfort and anxiety/depression. Each dimension has 5 levels: no problems, slight problems, moderate problems, severe problems and extreme problems. The EQ VAS can be used as a quantitative measure of health outcome that reflects the patient's health condition. The EQ-5D-5L has been shown to have high responsiveness in patients. It will ensure that studies are undertaken to a high standard, a consistent study design and methods for collecting the health-state values [19].

\section{Methods/design}

\section{Aims and objectives}

This study aims to evaluate the therapeutic effect of Tui-na together with the oral DHJSD on the treatment of KOA in Hong Kong. The study will examine whether the proposed combined therapy can reduce pain intensity and improve knee function for subjects with KOA. The working hypothesis of this study is that application of Tui-na together with the oral administration of DHJSD is effective and safe in the treatment of KOA.

\section{Design and setting}

This will be a prospective, randomized wait-list controlled trial in subjects with KOA. After 2 weeks screening period, eligible subjects will be randomly assigned to the treatment group and wait-list control group in a 1:1 ratio. The total study period will be 10 weeks. The study flowchart is shown in Figure 1 . The study will be conducted at the Yan Oi Tong - The Chinese University of Hong Kong Chinese Medicine Clinic cum Training and Research Centres (in Tuen Mun and Islands Districts), Hong Kong SAR, China.

\section{Inclusion Criteria}

Subjects will be recruited for the study if they meet all of the following criteria:

1. Either gender;

2. $\geq 50$ years of age;

3. Meet the criteria of KOA according to the American College of Rheumatology:

a. Knee pain, and

b. Any 4 of the following:

- $\geq 50$ years of age;

- Less than 30 minutes of morning stiffness; 
- Crepitus on active motion;

- Bony tenderness;

- Bony enlargement;

- No palpable warmth of synovium; and

- Osteophytes (based on radiographic findings).

4. With WOMAC score $\geq 39$,

5. Able to sign the written informed consent form (for illiterate subjects, their next-of-kin or an impartial witness will sign with subjects' permission).

\section{Exclusion criteria}

Subjects will be excluded if they possess any of the following criteria:

1. Known knee pain caused by infection, malignant or autoimmune diseases;

2. Knee surgery or arthroscopy in the past year;

3. Chondroprotective or intra-articular injection in the past 4 months;

4. Systemic corticosteroid treatment in the past 4 months;

5. Currently taking anticoagulants, antiplatelets, psychiatric drugs, hormones, antiarrhythmic drugs or diuretics drugs;

6. Uncontrolled hypertension;

7. Local antiphlogistic treatment, acupuncture, physiotherapy in the past 2 weeks;

8. Known history of mental disorder;

9. Known history of serious acute organic disease;

10. Renal or liver function impairment; and

11. Known allergy to or have drug interaction with the study herb.

\section{Recruitment}

All subjects will be recruited from the general public via daily outpatient services and advertisements on various social media. Subjects interested in participating in the study will be referred to or self-approach the study sites to undergo eligibility assessment. They will be prescreened through a telephone interview. Potential subjects then will have a face-to-face interview to confirm eligibility. During the interview, assessors will explain the overall objectives and nature of the study, describe the informed consent process, and assess the subjects' eligibility. The schedule of enrollment, interventions and assessments is shown in Table 1.

\section{Follow-up visits}

All subjects will be assessed using Chinese medicine theory by the registered Chinese medicine practitioners (RCMPs). The eligible subjects will be randomized into two groups, i.e., treatment group 
which will receive the standardized Tui-na and DHJSD, and the wait-list control group which will receive Tui-na alone during the study period. Subjects in the treatment group will undergo 8 sessions of 20 minutes Tui-na therapy, administered over 4 weeks and take study medication twice daily concomitantly for 4 weeks. The wait-list control group will receive standardized Tui-na therapy in the same manner with the treatment group but will not be given the DHJSD. Compensatory medication may be given after the completion of follow-up assessments at subject's discretion. Both groups will be assessed at day 0 (baseline), day $14 \pm 3$ days (week 2), and day $28 \pm 4$ days (week 4), and further followed up on day $56 \pm 4$ days (at week 8 , i.e., 4 weeks after treatment completion).

All outcome assessments will be done at baseline ( 0 week), 2 weeks, 4 weeks after randomization and follow-up (4 weeks after treatment completion).

If the subject has bilateral KOA and both knees fulfil the eligibility criteria, the WOMAC index and EQ-5D$5 \mathrm{~L}$ are used for the same knee throughout the whole study period. If the subject is illiterate, the questionnaire content will be explained by trained colleagues who are unrelated to the study.

Before the commencement of the trial, we will assess the subjects' vital signs, blood pressure and conduct laboratory tests on complete blood count, renal and liver functions. Post-study blood test will be done on day $28 \pm 4$ days (week 4 ) after randomization as a mean for the subjects' safety monitoring.

For safety concern, we will record medical history, adverse events during the treatment and follow-up period, and give appropriate treatment or referral if needed. Previous researches reported that DHJSD may cause gastrointestinal symptoms, including nausea and diarrhoea [20,21], however, serious adverse events were not found [22]. Treatment will be suspended immediately should treatment-related serious adverse events occur. Further assessment will be needed to decide whether the trial should be suspended. There will have a designated telephone hotline for adverse event reporting. Subjects can call the hotline during office hours but are advised to attend Emergency Department at the nearest hospital during nonoffice hours if severe adverse event is found.

\section{Randomization and blinding}

This study is designed as a randomized, wait-list controlled trial. Eligible subjects will be randomly allocated to one of the two groups: treatment group and wait-list control group. Random Allocation Software (version 1.0.0, Isfahan, Iran) will be used to generate a randomization scheme. An independent colleague who is not involved in the study will conduct the randomization process. The allocations will be concealed and sequentially numbered, and put in opaque, sealed envelopes. The information on the allocation list will remain strictly confidential.

\section{Background of the practitioners}

The study treatments will be performed by Registered Chinese Medicine Practitioners (RCMPs) in Hong Kong. The RCMPs can be both the assessors and Tui-na practitioners. The RCMPs will be responsible for consultation, assessment and prescription of study medication while the Tui-na practitioners will perform 
the Tui-na therapy alone. A CMP can be the assessor or Tui-na practitioner for different subjects; however, the role for each CMP will be fixed to avoid bias. For administering Tui-na therapy, responsible Tui-na CMP practitioners must have at least 2 years of clinical experience. The pre-trial training workshops on Tui-na therapy will be held to ensure standardization of Tui-na manipulation and procedures. Tui-na practitioners will be given a standardized script and instructed not to communicate with the subjects about the treatment effects.

\section{Intervention:}

\section{Tui-na manipulation [23]}

At the beginning, the subject will take a supine position. The trained Tui-na practitioner will apply rolling manipulation from the quadriceps femoris to the knee cap, and pressing, kneading both sides of knee cap for 5 minutes. Pressing and kneading Xuehai (SP10), Liangqiu (ST34), Heding (Ex-LE2), Dubi (ST35), Neixiyan (Ex-LE4) and Yanglingquan (GB34) for 1 minute each.

Subsequently, the subject will take a prone position. The Tui-na practitioner will apply rolling manipulation from the bottom of the thigh to the top of the calf for 5 minutes. Weizhong (BL40) and Chengshan (BL57) are pressed for 1 minute each.

Next, the patient takes a supine position, with the affected knee and hip flexed. The Tui-na practitioner supports the upper part of the knee to fix it with one hand and holds the heel with the other hand to rotate the knee joint clockwise, then counterclockwise for 5 times each.

The Tui-na practitioner will then hold the ankle and lift the treated extremity. The lower extremity should be raised to form a 30 degrees angle between the extremity and the bed. While pulling with slight force, shake constantly up and down in a small amplitude for 10 seconds.

Finally, the Tui-na practitioner will rub the sides of the knee joint, the edge of knee cap for 15 seconds. The names and locations of acupoints are listed in Table 2.

\section{Study Medication}

Subjects in the treatment group will be prescribed and dispensed with oral study medicine by the RCMPs. This study medication is Du-Huo-Ji-Sheng Decoction (DHJSD) [24] in concentrated Chinese medicine granule form. Each daily dose of DHJSD consists of Du-huo (AngelicaePubescentis Radix) 2g, Sang-jisheng (TaxilliHerba) 3g, Du-zhong (Eucommiae Cortex) 2g, Niu-xi (AchyranthisBidentatae Radix) 2.4g, Xixin (Asari Radix et Rhizoma) 0.2g, Qin-jiao (GentianaeMacrophyllae Radix) 2g, Fu-ling (Poria) 3g, Fangfeng (Saposhnikoviae Radix) 2g, Chuan-xiong (Chuanxiong Rhizoma) 1.2g, Dang-shen (Codonopsis Radix) 3g, Gan-cao (Glycyrrhizae Radix et Rhizoma) 1.2g, Bai-shao (Paeoniae Radix Alba) 2.4g, Di-huang (Rehmanniae Radix) 3g, Ji-xue-teng (Spatholobi Caulis) 2.4g [9] , Cu-yan-hu-suo (Corydalis Rhizoma) 0.6g [9]. The study medication will be taken twice daily for 4 consecutive weeks. 
The study medication will be adjusted according to subjects' clinical manifestation pattern based on Chinese medicine theory. If the subject manifests excessive dampness pattern, Fang-ji (StephaniaeTetrandrae Radix) 2g, Yi-yi-ren (Coicis Semen) 3g and Cang-zhu (AtractylodisRhizoma) 1.8g will be added [24]. If the participant manifests a blood stasis pattern, Tao-ren (Persicae Semen) 2g, Honghua (CarthamiFlos) $1.2 \mathrm{~g}$ will be added instead [25]. Prescription of the core formula and the addition of herbs are listed in Table 3.

The granules used in this study will be manufactured under Good Manufacturing Practice (GMP) standard. Subjects will be explained clearly on how to administer and consume the study medicine. An instruction sheet (Appendix I) will be delivered to the subjects together with the pre-packed granule bags. This includes the storage and administering information of the medicine, as well as its common sideeffects. Since the study medication will be prescribed and dispensed by RCMPs, it is not categorized as proprietary Chinese medicine, therefore the trial does not require Certificate for Clinical Trial and Medicinal Test.

\section{Permitted and prohibited concomitant treatments}

All Chinese herbal medicine or Chinese medicine therapies such as acupuncture \& moxibustion other than study treatment will be prohibited.

\section{Study termination}

Subject's participation would be terminated if he/she has:

1) developed severe adverse event;

2) hypersensitivity towards study treatment;

3) participated in another interventional research project;

4) life-threatening disease; or

5) decided to withdraw from the trial.

The whole research would be terminated under the following circumstances:

1) presence of serious adverse events related to the study medication; or

2) completion of all follow-up assessments.

\section{Outcome measurements}


The primary outcome measure will be rated using the WOMAC in Cantonese version $[15,16]$ (Appendix II) at 4 weeks after randomization. The WOMAC is a self-administered 3-dimensional questionnaire that assesses pain, stiffness and physical functional disability in subjects with KOA using a series of 24 questions. It is a 5 -point scale from 0 to $4(0=$ none, $1=$ mild, $2=$ moderate, $3=$ severe, $4=$ extreme $)$.

\section{Secondary outcome measure}

Secondary outcome measures include WOMAC in Cantonese version undertaken at 2 weeks and 8 weeks after randomization. The health-related quality of life using the EQ-5D-5L in Chinese version [17-19] (Appendix III) will also be used. Outcomes will be documented at 2 weeks, 4 weeks after randomization and follow-up (8 weeks after randomization). The patient is asked to indicate his/her health state by ticking the box in EQ-5D next to the most appropriate statement in each of the five dimensions. The EQ VAS records the patient's self-rated health on a vertical visual analogue scale, where the endpoints are labelled 'The best health you can imagine' and 'The worst health you can imagine'.

\section{Known herb-drug interaction and adverse events}

For the herb drug interaction, Du-huo (AngelicaePubescentis Radix) may pose moderate risk to subjects taking cytochrome P450 1A2 (CYP1A2) substrates. Dang-shen (Codonopsis Radix) and Bai-shao (Paeoniae Radix Alba) may pose moderate risk to subjects taking anticoagulant or antiplatelet drugs. Besides, Bai-shao may pose moderate risk to subjects taking clozapine and phenytoin. Gan-cao (Glycyrrhizae Radix et Rhizoma) may pose moderate risk to subjects taking antiarrhythmic drugs, antihypertensive drugs, diuretics, positive inotropic drugs, corticosteroids, hypnotics, anxiolytics, estrogen and testosterone. It is also suggested that Gan-cao may pose major risk when it is concurrently used with warfarin. Di-huang (Rehmanniae Radix) may pose moderate risk to subjects taking antiplatelet drugs [26]. For other herbs used in this study, no clinical trials or case reports of suspected herb drug interaction were identified. In addition, the DHJSD may induce mild gastrointestinal discomfort such as vomiting and diarrhea, while Tui-na may induce mild local pain. All these adverse events are known and will usually disappear in a short period of time.

\section{Dropouts and withdrawals}

All dropouts and attrition during the courses of the study will be monitored, and the respective reasons for withdrawal recorded.

\section{Data management}

This is a 10-week clinical trial, in which subjects must take treatment for 4 weeks (during $2^{\text {nd }}-6^{\text {th }}$ weeks), attend four assessment visits, complete a set of questionnaires, and stop taking other medications to avoid interference of the trial. All collected information will be input into a designed database with password protection. Study documents will be kept for 7 years. We will explain the details of the study schedule, treatment, risk and benefits of the study, the responsibility of both assessors and the subjects 
during the consent process. In addition, before each visit, the delegated colleague will contact the subject to re-confirm the schedule. If a subject shows signs of wanting to withdraw, we would try to understand the underlying reasons and to explore the possibility to continue with the study.

\section{Sample size calculation}

The sample size calculation is based on the primary outcome i.e., the WOMAC. We assumed Tui-na combined with Chinese herbal medicine could have a 20-point superior than the wait-list control group at week 4. According to the previous study, the Tui-na could decrease WOMAC by 17.44 , with the standard deviation of 23.61 [27]. The $\mu$ sample size could be calculated according to the following formula

$$
n=\frac{2 \sigma^{2}\left(t_{\alpha}+t_{\beta}\right)^{2}}{\left(\mu_{1}-\mu_{2}\right)^{2}}
$$

The $\mu 1-\mu 2$ is the difference in the means, which is 2.616. The $\sigma$ was 23.61 . We define $\alpha$ as 0.05 and $\beta$ as 0.1 , the ta and $t \beta$ are 1.96 and 1.282 respectively. The sample size for each group is 29 . Considering $20 \%$ drop-out, the total sample size will be 70 .

\section{Statistical analysis}

All efficacy and safety analyses will be conducted according to the intention-to-treat (ITT) principle. Missing values will be imputed by the last-observation-carried forward method. The statistical analysis will be performed using the Statistical Packages for the Social Sciences (SPSS) for Windows, version 22.0. Statistical significance is defined as a two-sided $p$ value of $<0.05$. Baseline characteristics will be reported as mean $\pm S D$. Comparisons between groups will be conducted by using an analysis of covariance (ANCOVA) with baseline as a covariate. All items and subscales will be compared between groups for each 4-week period using ANCOVA, with treatment group as a factor in the model and baseline as the covariate. The changes in scores from baseline to the end-point of treatment will be tested using ANOVA. Within-group differences will be assessed using a paired t-test for normally distributed data and a Wilcoxon signed-rank test for non-normally distributed data.

\section{Study monitoring}

A dedicated team from the Hospital Authority Chinese Medicine Department (HACMD) that is completely independent in the running of the study and has no competing interests will be responsible for monitoring the study progress. Pre-trial and post-trial onsite inspections and quarterly monitoring meetings will be held to oversee the progress of the study and ensure it is conducted, recorded, and reported in accordance with the protocol, Good Clinical Practice (GCP) guidelines, and applicable regulatory requirements. Although a separate data monitoring committee will not be established, the HACMD will serve this function to ensure scientific validity, scientific integrity, and data accuracy through the regular monitoring of this clinical trial. 


\section{Ethical consideration and dissemination of information}

The ethical validity of the study was approved by the Joint Chinese University of Hong Kong - New Territories East Cluster Clinical Research Ethics Review Committee (CREC Ref. No. 2019.538). Any modifications to the protocol are reported to this committee, and amendment approval should be obtained before any changes can take place. The written consent from individual subjects will be obtained voluntarily before the intervention. All patient data will be coded and anonymized when entered into the case report form. Only de-identified data will be collected and removed from the premises. We plan to publish the results of this study in peer-reviewed journals or academic conference proceedings.

\section{Discussion}

Subjects who experience knee problems often suffer from pain and functional limitations. The prevalence of disabling knee pain will increase as the population ages. Tui-na has been used to treat pain and functional limitations of knee pain for many years with positive results. In order to maximize the effectiveness of the Tui-na, we add other therapies to create a synergistic effect on the KOA treatment. Since DHJSD is a widely used traditional Chinese herbal formula for the treatment of arthritis in China $[8,9]$, we aim to evaluate the therapeutic effect of the proposed combined therapy on the treatment of KOA. However, because of the methodology limitations in the previous studies, little scientific evidence exists to support the effectiveness of the methods. Therefore, we designed this study with an adequate methodology that follows international guidelines and uses validated assessment tools.

This study is a prospective, randomized, wait-list controlled trial with an aim to investigate the therapeutic effect of the combined Tui-na therapy and oral DHJSD for treating subjects with KOA. There is a limitation of the study in that a high risk of bias may exist regarding blinding as we used a waiting list as the control instead of a sham procedure. Owing to the nature of the Tui-na therapy, neither the study subjects nor the assessors can be blinded to the allocation and treatment stages.

This rigorously designed clinical trial will provide evidence-based knowledge on whether Tui-na plus oral DHJSD is efficacious and safe in relieving pain, improving knee function and quality of life among older adults with KOA in Hong Kong. More importantly, the results obtained in the proposed study will provide valuable information and a solid foundation for future larger-scale clinical studies on the same topic.

\section{Trial status}

This protocol was Version 2 written on 11 June 2020. The study will commence once we receive ethical approval. Subject recruitment will start from December 2020 and is expected to be completed before June 2022.

\section{Abbreviations}


CREC: Clinical Research Ethics Review Committee; CYP1A2: Cytochrome P450 1A2; DHJSD: Du-Huo-JiSheng Decoction; EQ VAS: EQ visual analogue scale; EQ-5D-5L: the 5-level EQ-5D version; GCP: Good Clinical Practice; GMP: Good Manufacturing Practice; HACMD: Hospital Authority Chinese Medicine Department; ITT: intention-to-treat; KOA: Knee osteoarthritis; RCMPs: Registered Chinese medicine practitioners; SD: standard deviation; SPSS: Statistical Packages for the Social Sciences; TKR: Total Knee Replacement; WOMAC: Western Ontario and McMaster University Osteoarthritis Index.

\section{Declarations}

\section{Acknowledgement}

The authors wish to acknowledge the support by the Hospital Authority Chinese Medicine Department, Hong Kong.

\section{Authors' contributions}

ZXL is the Principal Investigator and project leader. KHC and ZXL conceived and designed the study. JYLC, KLC, HYL, KMC, KC, HFP and TS advised and contributed to the trial design. KHC sought funding. KHC, JYLC, KLC and ZXL obtained ethical approval. KHC drafted the study protocol. JYLC, KLC, BFLN and ZXL provided expert advice and critical comments on the protocol. All authors read and approved the final manuscript.

\section{Funding}

This study is supported by a research grant from the "Hospital Authority Chinese Medicine Research Practical Training Programme 2018/19" launched by the Hospital Authority Chinese Medicine Department, Hong Kong SAR. The funder had no role in the study design; the collection, management, analysis, and interpretation of the data; the writing of the report; or the decision to submit the manuscript for publication; or authority over any of these activities.

\section{Availability of data and materials}

The datasets used and/or analysed during the current study are available from the corresponding author on reasonable request.

\section{Ethics approval and consent to participate}

The study protocol was approved by The Joint Chinese University of Hong Kong - New Territories East Cluster Clinical Research Ethics Committee (Ref. No.: 2019.538). Any changes to the protocol will be reported to the Committee. This study is conducted in compliance with the Declaration of Helsinki and the ICH-GCP. Written informed consent will be obtained from all study participants in the trial before enrolment.

\section{Consent for publication}


Not applicable

\section{Competing interests}

The authors declare that they have no competing interests.

\section{References}

1. Felson DT, Naimark A, Anderson J, Kazis L, Castelli W, Meenan RF. The prevalence of knee osteoarthritis in the elderly. the Framingham osteoarthritis study. Arthritis Rheum. 1987;30(8):914-8.

2. The Census and Statistics Department (C\&SD). Hong Kong Population Projections 2017-2066. Hong Kong, 2017. Available at: https://www.censtatd.gov.hk/media_workers_corner/pc_rm/hkpp2017_2066/index.jsp

3. Kotlarz H, Gunnarsson CL, Fang H, Rizzo JA. Insurer and out-of-pocket costs of osteoarthritis in the US: Evidence from national survey data. Arthritis Rheum. 2009;60(12):3546-53.

4. Næsdal J, Brown K. NSAID-associated adverse effects and acid control aids to prevent them: A review of current treatment options. Drug Saf. 2006;29(2):119-32.

5. Hospital Authority. Waiting Time for Total Joint Replacement Surgery. 2018. Available at: https://www.ha.org.hk/visitor/ha_visitor_text_index.asp?Parent_ID=214172\&Content_ID=221223.

6. Pain IA for the S of. 2017 Global Year Against Pain After Surgery. 2017. Available from: file:///E:/Chronic Postsurgical Pain/2017 Global Year Against Pain After Surgery - IASP.htm.

7. Bender T, Nagy G, Barna I, Tefner I, Kádas É, Géher P. The effect of physical therapy on betaendorphin levels. Eur J Appl Physiol. 2007;100(4):371-82.

8. Zhao J, Zha Q, Jiang M, Cao H, Lu A. Expert consensus on the treatment of rheumatoid arthritis with Chinese patent medicines. J Altern Complement Med. 2013;19(2):111-8.

9. Chen FP, Chang CM, Hwang SJ, Chen YC, Chen FJ. Chinese herbal prescriptions for osteoarthritis in Taiwan: analysis of national health insurance dataset. BMC Complement Altern Med. 2014;14:91.

10. Lv S, Zhou JJ, Xie XT, et al. Mechanism research and clinical application of Duhuo Jisheng Decoction in treating knee osteoarthritis. Global Chin Med. 2015;8(9):1149-52.

11. Ying YS, Hu YJ. Observation on the efficacy and safety of applying Duhuo Jisheng Decoction in treating knee osteoarthritis. Chin Comm Physician. 2011, 36(13): 177-8.

12. Du-Huo-Ji-Sheng-Tang attenuates inflammation of TNF-Tg Mice related to promoting lymphatic drainage function. Evid Based Complement Alternat Med. 2016;2016:7067691.

13. American College of Rheumatology Subcommittee on Osteoarthritis Guidelines. Recommendations for the medical management of osteoarthritis of the hip and knee:2000 update. Arthritis Rheum. 2000;43:1905-15.

14. Altman R, Asch E, Bloch D, Bole G, Borenstein D, Brandt K, Christy W, Cooke TD, Green R, Hochberg M. Development of criteria for the classification and reporting of osteoarthritis.: classification of 
osteoarthritis of the knee: diagnostic and therapeutic criteria committee of the American rheumatism association. Arthritis Rheum.1986;29:1039-1049.

15. Ackerman H. Western Ontario and McMaster Universities Osteoarthritis Index (WOMAC). Aus J Phys. 2009;55:213.

16. Zhang W, Moskowitz RW, Nuki G, Abramson S, Altman RD, Arden N, Bierma-Zeinstra S, Brandt KD, Croft P, Doherty $\mathrm{M}$, et al. OARSI recommendations for the management of hip and knee osteoarthritis, part II: OARSI evidence-based, expert consensus guidelines. Osteoarthr Cartil. 2008;16(2):137-62.

17. Devlin N, Shah K, Feng Y, Mulhern B, van Hout B. Valuing health-related quality of life: an EQ-5D-5L value set for England.Office of Health Economics: London, UK; 2016.

18. Herdman M, Gudex C, Lloyd A, Janssen M, Kind P, Parkin D, Bonsel G, Badia X. Development and preliminary testing of the new five-level version of EQ-5D (EQ-5D-5L). Qual Life Res. 2011;20:172736.

19. Tidermark J, Bergström G, Svensson O, Törnkvist H, Ponzer S. Responsiveness of the EuroQol (EQ 5D) and the SF-36 in elderly patients with displaced femoral neck fractures. Qual Life Res. 2003;12(8):1069-79.

20. Cao GP, Hu JX, Wang CF. Clinical observation of DuhuoJisheng decoction combined with sodium hyaluronate injection in intra-articular cavity injection in the treatment of knee osteoarthritis. Zhongguo Shi Yan Fang Ji Xue Za Zhi. 2013;19:305-8.

21. Zhang L, Sun DY. Clinical observation of diacerein combined with DuohuoJisheng decoction in the treatment of middle and aged people with knee osteoarthritis. Zhongguo Shi Yan Fang Ji Xue Za Zhi. 2013;19:299-302.

22. Chen B, Zhan H, Marszalek J, Chung M, Lin X, Zhang M, Pang J, Wang C. Traditional Chinese medications for knee osteoarthritis pain: A Meta-Analysis of randomized controlled trials. Am J Chin Med. 2016;44(4):677-703.

23. Yan JT. "Manipulation". China: China Press of Traditional Chinese Medicine. 1996.

24. Duan FJ. "Prescription Science". China: Shanghai Science \& Technology Publisher. 1995.

25. He MT, Li Y, Shao M, Liang ZJ, Huang WJ, Xu WJ. The Clinical Efficacy of Traditional Hinese Drug for Integrating Manipulation to Treat Knee Osteoarthtltis. Chin J Trad Med Traum \& Orthop. 2007;15(8):25-8.

26. Natural Medicines, The Authority of Integrative Medicine. 2019. Available at: https://naturalmedicines.therapeuticresearch.com.

27. Perlman Al, Sabina A, Williams AL, Njike VY, Katz DL.Massage therapy for osteoarthritis of the knee: a randomized controlled trial. Arch Intern Med. 2006;166(22):2533-8.

\section{Tables}

Table 1 SPIRIT Schedule of enrolment, interventions, and assessments 


\begin{tabular}{|c|c|c|c|c|c|}
\hline & \multicolumn{5}{|c|}{ STUDY PERIOD } \\
\hline & \multirow{2}{*}{$\begin{array}{c}\text { Enrolment } \\
-t^{t}\end{array}$} & \multirow{2}{*}{$\begin{array}{c}\text { Randomization } \\
0\end{array}$} & \multicolumn{2}{|c|}{$\begin{array}{c}\text { Treatment } \\
\text { (weeks) }\end{array}$} & \multirow{2}{*}{$\begin{array}{c}\begin{array}{c}\text { Follow- up } \\
\text { (weeks) }\end{array} \\
8\end{array}$} \\
\hline TIMEPOINT** & & & 2 & 4 & \\
\hline \multicolumn{6}{|l|}{ ENROLMENT: } \\
\hline \multirow{2}{*}{$\begin{array}{l}\text { Eligibility screen } \\
\text { Informed consent }\end{array}$} & $\mathrm{x}$ & & & & \\
\hline & $\mathrm{x}$ & & & & \\
\hline Laboratory test & $\mathrm{x}$ & & & & \\
\hline CMP assessment & & $\mathrm{x}$ & $\mathrm{x}$ & $\mathrm{x}$ & $\mathrm{X}$ \\
\hline Allocation & & $\mathrm{x}$ & & & \\
\hline \multicolumn{6}{|l|}{ INTERVENTIONS: } \\
\hline \multicolumn{6}{|l|}{ Tui-na } \\
\hline \multicolumn{6}{|l|}{ Study herb medication } \\
\hline & & & & & \\
\hline \multicolumn{6}{|l|}{ ASSESSMENTS: } \\
\hline WOMAC & & $\mathrm{x}$ & $\mathrm{x}$ & $\mathrm{X}$ & $\mathrm{x}$ \\
\hline EQ-5D-5L & & $\mathrm{x}$ & $\mathrm{x}$ & $\mathrm{x}$ & $\mathrm{x}$ \\
\hline \multicolumn{6}{|l|}{ EQ VAS } \\
\hline $\begin{array}{r}\text { Adverse event } \\
\text { assessment }\end{array}$ & & & $\mathrm{x}$ & $\mathrm{X}$ & $\mathrm{X}$ \\
\hline
\end{tabular}

*The duration between initial screening and randomisation will vary depending on the time it takes a participant to complete baseline measures. However, we anticipate that all participants will be randomised within 2 weeks of their initial screening appointment

Table 2 Acupoints used in the study* 


\begin{tabular}{|c|c|c|c|}
\hline $\begin{array}{l}\text { Name of } \\
\text { acupoints }\end{array}$ & Areas & $\begin{array}{l}\text { Special } \\
\text { qualification }\end{array}$ & Indications \\
\hline $\begin{array}{l}\text { Xuehai } \\
\text { (SP10) }\end{array}$ & $\begin{array}{l}\text { When the knee is flexed, } 2 \text { cun } \\
\text { above the medical superior } \\
\text { border of the patella on the } \\
\text { bulge of the medial portion of } \\
\mathrm{m} \text {. quadriceps femoris }\end{array}$ & - & $\begin{array}{l}\text { Irregular menstruation, heavy } \\
\text { uterine bleeding, amenorrhea; } \\
\text { urticaria, eczema, erysipelas; } \\
\text { abdominal distension, diarrhea, } \\
\text { jaundice; abdominal pain, } \\
\text { difficulty in micturition, edema; } \\
\text { pain and swelling in the knees }\end{array}$ \\
\hline $\begin{array}{l}\text { Liangqiu } \\
\text { (ST34) }\end{array}$ & $\begin{array}{l}\text { On the line connecting the } \\
\text { anterior superior iliac spine and } \\
\text { the lower lateral border of the } \\
\text { patella, 2cun above the upper } \\
\text { lateral border of the patella }\end{array}$ & $\begin{array}{l}\text { Xi- Cleft } \\
\text { Point }\end{array}$ & $\begin{array}{l}\text { Stomach pain; knee pain, } \\
\text { atrophy and paralysis of the } \\
\text { legs; mastitis }\end{array}$ \\
\hline $\begin{array}{l}\text { Heding (Ex- } \\
\text { LE2) }\end{array}$ & $\begin{array}{l}\text { Above the knee, in the } \\
\text { depression of the midpoint of } \\
\text { the superior patellar border }\end{array}$ & - & $\begin{array}{l}\text { Knee pain, weakness of the leg } \\
\text { and foot, and palsy }\end{array}$ \\
\hline Dubi (ST35) & $\begin{array}{l}\text { When the knee is flexed, at the } \\
\text { lower border of the patella, in } \\
\text { the depression lateral to the } \\
\text { patella ligament }\end{array}$ & - & $\begin{array}{l}\text { Swelling and pain in the knees, } \\
\text { difficulty in flexing and } \\
\text { extending the knees and beriberi }\end{array}$ \\
\hline $\begin{array}{l}\text { Neixiyan (Ex- } \\
\text { LE4) }\end{array}$ & $\begin{array}{l}\text { In the depression medial to the } \\
\text { patellar ligament when the knee } \\
\text { is flexed }\end{array}$ & - & $\begin{array}{l}\text { Pain in the knee, atrophy or } \\
\text { paralysis of the lower limbs }\end{array}$ \\
\hline $\begin{array}{l}\text { Yanglingquan } \\
\text { (GB34) }\end{array}$ & $\begin{array}{l}\text { In the depression anterior and } \\
\text { inferior to the small head of the } \\
\text { fibula }\end{array}$ & $\begin{array}{l}\text { He-Sea } \\
\text { Point; Lower } \\
\text { He-Sea } \\
\text { Point of the } \\
\text { Gallbladder; } \\
\text { One of the } \\
\text { Eight } \\
\text { Influential } \\
\text { Points }\end{array}$ & $\begin{array}{l}\text { Jaundice, bitter taste in mouth, } \\
\text { hiccups, vomiting; pain in the } \\
\text { hypochondriac region; swelling } \\
\text { and pain of the knee, atrophy or } \\
\text { paralysis in the lower limbs }\end{array}$ \\
\hline $\begin{array}{l}\text { Weizhong } \\
\text { (BL40) }\end{array}$ & $\begin{array}{l}\text { On the midpoint of the } \\
\text { transverse crease of the } \\
\text { popliteal fossa, between the } \\
\text { tendons of the biceps femoris } \\
\text { and semitendinous }\end{array}$ & $\begin{array}{l}\text { He-Sea } \\
\text { Point; Lower } \\
\text { He-Sea } \\
\text { Point of the } \\
\text { Bladder }\end{array}$ & $\begin{array}{l}\text { Lumbar pain, spasm of the } \\
\text { popliteal tendons, weakness or } \\
\text { paralysis in the lower limbs; } \\
\text { difficulty in urination, enuresis; } \\
\text { erysipelas, urticaria,, furuncles }\end{array}$ \\
\hline $\begin{array}{l}\text { Chengshan } \\
\text { (BL57) }\end{array}$ & $\begin{array}{l}\text { In the centre of the posterior } \\
\text { aspect of the lower leg, between } \\
\text { Weizhong (BL40) and Kunlun } \\
\text { (BL60), in the triangle } \\
\text { depression formed below the } \\
\text { bellies of the gastrocnemius } \\
\text { muscle when the foot is } \\
\text { stretched }\end{array}$ & - & $\begin{array}{l}\text { Pain and spasm in the lumbar } \\
\text { region and legs; haemorrhoids, } \\
\text { constipation }\end{array}$ \\
\hline
\end{tabular}

*According to "Acupuncture and Moxibustion" edited by Shi XM (China Press of Traditional Chinese Medicine, 2002) 
Table 3 The composition of the DHJSD and the additional herbs* 
Concentrated

Chinese Medicine

granules

Concentration 1:5
Corresponding dose of raw herb
Highest dosage recommended by Chinese Pharmacopeia

The Basic DHJSD

Du-huo (Angelicae

$2 g$

$10 \mathrm{~g}$

$10 \mathrm{~g}$

Pubescentis Radix)

$3 g$

$15 \mathrm{~g}$

$15 g$

Du-zhong (Eucommiae

Cortex)

Niu-xi (Achyranthis

Bidentatae Radix)

$2 \mathrm{~g}$

$10 \mathrm{~g}$

$10 \mathrm{~g}$

Xi-xin (Asari Radix et

Rhizoma)

$2.4 \mathrm{~g}$

$12 \mathrm{~g}$

$12 \mathrm{~g}$

$0.2 \mathrm{~g}$

$1 \mathrm{~g}$

$1 \mathrm{~g}$

Qin-jiao (Gentianae

Macrophyllae Radix)

$2 \mathrm{~g}$

$10 \mathrm{~g}$

$10 \mathrm{~g}$

Fu-ling (Poria)

$3 \mathrm{~g}$

$15 \mathrm{~g}$

$15 g$

Fang-feng (Saposhnikoviae $\quad 2 \mathrm{~g}$

Radix)

Chuan-xiong (Chuanxiong

Rhizoma)

$1.2 \mathrm{~g}$

$10 \mathrm{~g}$

$10 \mathrm{~g}$

Dang-shen (Codonopsis

Radix)

$3 g$

$15 g$

$30 \mathrm{~g}$

Gan-cao (Glycyrrhizae Radix $\quad 1.2 \mathrm{~g}$

$6 \mathrm{~g}$

$10 \mathrm{~g}$

et Rhizoma)

Bai-shao (Paeoniae Radix

$2.4 \mathrm{~g}$

$12 \mathrm{~g}$

$15 g$

Alba)

Di-huang (Rehmanniae

Radix)

Ji-xue-teng (Spatholobi

Caulis)

$3 g$

$15 \mathrm{~g}$

$15 g$

Cu-yan-hu-suo (Corydalis

Rhizoma)

$2.4 \mathrm{~g}$

$12 \mathrm{~g}$

$15 \mathrm{~g}$

$0.6 \mathrm{~g}$

$3 g$

$3 g$

Addition of herbs to subjects

with excess dampness

pattern

Fang-ji (Stephaniae

Tetrandrae Radix)

$2 \mathrm{~g}$

$10 \mathrm{~g}$

$10 \mathrm{~g}$ 


\begin{tabular}{|llcc|}
\hline Yi-yi-ren (Coicis Semen) & $3 \mathrm{~g}$ & $15 \mathrm{~g}$ & $30 \mathrm{~g}$ \\
\hline $\begin{array}{l}\text { Cang-zhu (Atractylodis } \\
\text { Rhizoma) }\end{array}$ & $1.8 \mathrm{~g}$ & $9 \mathrm{~g}$ & $9 \mathrm{~g}$ \\
\hline $\begin{array}{l}\text { Addition of herbs to subjects } \\
\text { with blood stasis pattern }\end{array}$ & & & \\
\hline Tao-ren (Persicae Semen) & $2 \mathrm{~g}$ & $10 \mathrm{~g}$ & $10 \mathrm{~g}$ \\
\hline Hong-hua (Carthami Flos) & $1.2 \mathrm{~g}$ & $6 \mathrm{~g}$ & $10 \mathrm{~g}$ \\
\hline
\end{tabular}

*According to

1. Duan FJ. "Prescription". Shanghai Science \& Technology Publisher, 1995.

2. Chen FP, Chang CM, Hwang SJ, Chen YC, Chen FJ. Chinese herbal prescriptions for osteoarthritis in Taiwan: analysis of national health insurance dataset. BMC Complement Altern Med. 2014;14:91.

3. He MT, Li Y, Shao M, Liang ZJ, Huang WJ, Xu WJ. The Clinical Efficacy of Traditional Hinese Drug for Integrating Manipulation to Treat Knee Osteoarthtltis. Chin J Trad Med Traum \& Orthop. 2007;15(8):25-8.

\section{Figures}




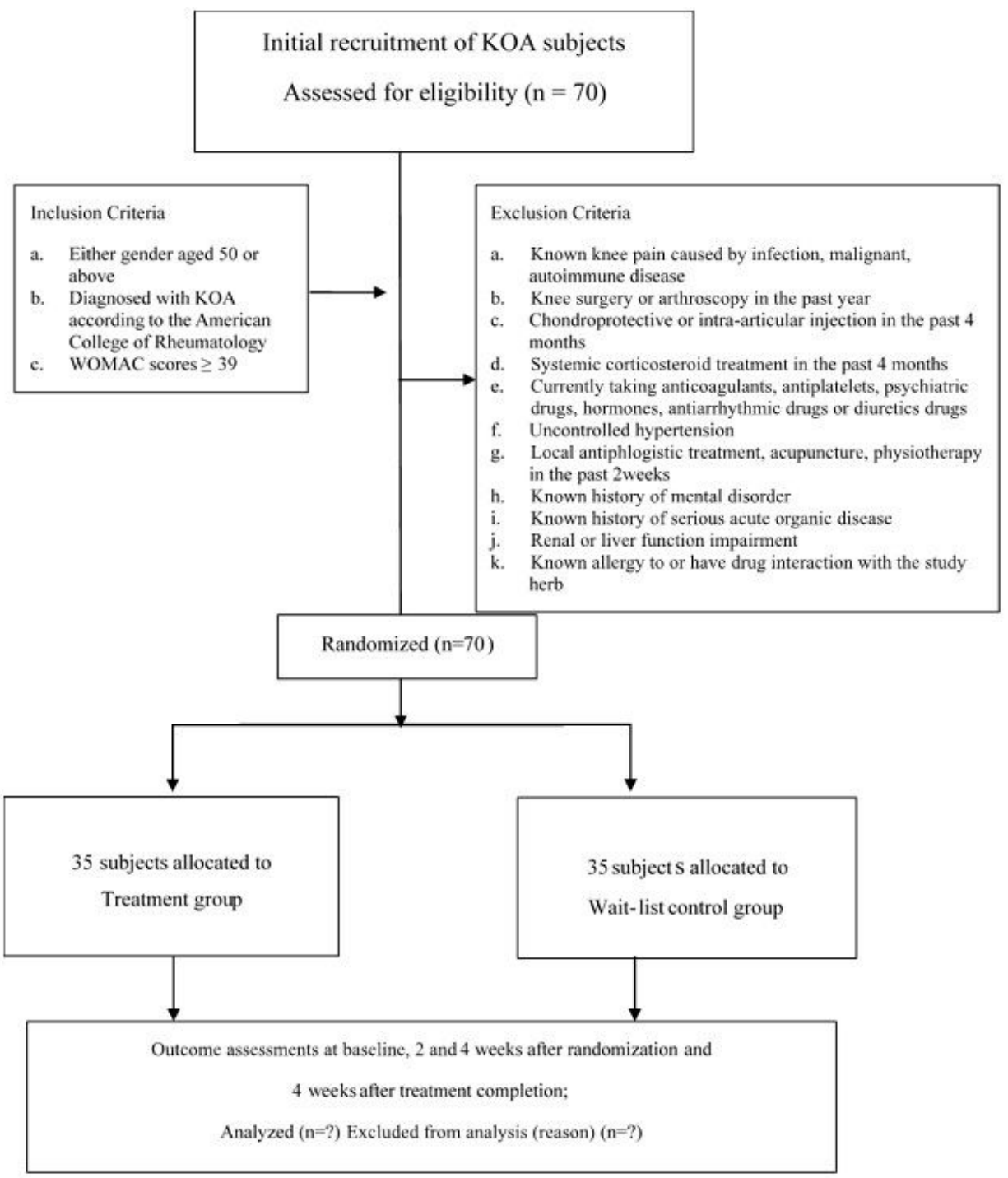

\section{Figure 1}

The study will be conducted at the Yan Oi Tong - The Chinese University of Hong Kong Chinese Medicine Clinic cum Training and Research Centres (in Tuen Mun and Islands Districts), Hong Kong SAR, China.

\section{Supplementary Files}


This is a list of supplementary files associated with this preprint. Click to download.

- Appendixltolll.docx

- SPIRITFillablechecklist15Aug2013.doc 